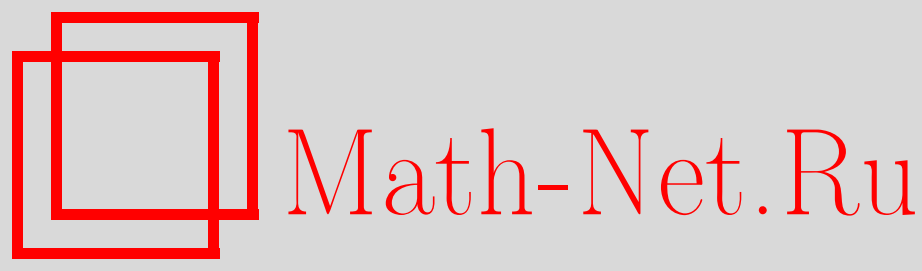

М. Э. Муминов, О бесконечности числа собственных значений на лакуне существенного спектра оператора Шредингера трех частиц на решетке, ТМФ, 2009, том 159, номер 2, 299-317

DOI: https://doi.org/10.4213/tmf6350

Использование Общероссийского математического портала Math-Net.Ru подразумевает, что вы прочитали и согласны с пользовательским соглашением http://www . mathnet.ru/rus/agreement

Параметры загрузки:

IP : 52.205 .19 .152

26 апреля 2023 г., 11:44:28

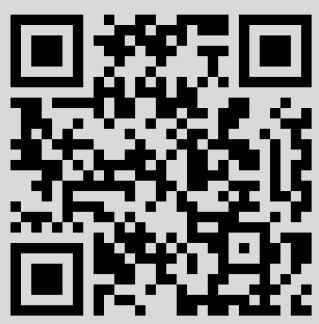




\title{
ФИЗИКА
}

Том 159, № 2

май, 2009

2009 г.

М. Э. Муминов*

\section{О БЕСКОНЕЧНОСТИ ЧИСЛА СОБСТВЕННЫХ ЗНАЧЕНИЙ НА ЛАКУНЕ СУЩЕСТВЕННОГО СПЕКТРА ОПЕРАТОРА ШРЕДИНГЕРА ТРЕХ ЧАСТИЦ НА РЕШЕТКЕ}

\begin{abstract}
Рассматривается система трех произвольных квантовых частиц на трехмерной решетке, взаимодействующих с помощью парных контактных потенциалов притяжения. Найдено условие появления лакуны существенного спектра и доказано существование бесконечного числа собственных значений в этой лакуне для гамильтониана соответствующей системы трех частиц.
\end{abstract}

Ключевые слова: трехчастичная система на решетке, оператор Шредингера, существенный спектр, дискретный спектр, компактный оператор.

\section{1. ВВЕДЕНИЕ}

Существование бесконечного числа собственных значений, накапливающихся к левому краю существенного спектра оператора Шредингера (эффект Ефимова [1]), изучалось в работах [2], [3]. Строгое математическое доказательство этого факта с помощью интегральных уравнений Фаддеева было впервые дано в работе [4]. Затем существование бесконечного числа связанных состояний для гамильтониана системы трех частиц, взаимодействующих с помощью парных контактных потенциалов притяжения установлено в работах [5], [6]. При нулевом значении полного квазиимпульса системы этот результат доказан в работах [7]-[9]. Конечность связанных состояний дискретного оператора Шредингера трех частиц доказана в работах [8], [9] при малых значениях полного квазиимпульса $K \neq 0$. Заметим, что в работах [9], [10] бесконечное число собственных значений появляется ниже существенного спектра, если два из трех двухчастичных подгамильтонианов имеют виртуальные уровни в нуле, а нуль является регулярной точкой для третьего гамильтониана, или все двухчастичные подгамильтонианы имеют виртуальные уровни в нуле.

Отметим, что непрерывный спектр трехчастичного (непрерывного) оператора Шредингера совпадает с полуосью $\left[-\kappa^{2}, \infty\right), \kappa \geqslant 0$ (см. [11]). В отличие от существенного спектра трехчастичного непрерывного оператора Шредингера, существенный спектр $\sigma_{\text {ess }}(H(K))$ трехчастичного дискретного оператора Шрединге-

${ }^{*}$ Самаркандский государственный университет им. Алишера Навои, Самарканд, Узбекистан. E-mail: mmuminov@mail.ru 
ра $H(K)$ может содержать лакуны, т.е. существует интервал $(a, b), \inf \sigma_{\mathrm{ess}}(H(K)) \leqslant$ $a<b \leqslant \sup \sigma_{\text {ess }}(H(K))$, такой, что $(a, b) \cap \sigma_{\text {ess }}(H(K))=\varnothing$.

В настоящей работе рассматривается система трех произвольных квантовых частиц на трехмерной решетке, взаимодействующих с помощью парных контактных потенциалов притяжения. Рассмотрен случай, когда два двухчастичных подгамильтониана имеют виртуальные уровни в нуле и нуль является регулярной точкой для третьего подгамильтониана, при этом существенный спектр оператора $H(\mathbf{0})$, $\mathbf{0}=(0,0,0)$, содержит лакуну $(b, 0)$. Для каждого действительного $z$, лежащего вне существенного спектра $H(K)$, построен самосопряженный компактный оператор $\mathbf{T}(z)$ такой, что точки разрыва функции $n(1, \mathbf{T}(\cdot))$ являются собственными значениями оператора $H(K)$, где $n(1, \mathbf{T}(z))$ - число собственных значений оператора $\mathbf{T}(z)$, лежащих правее единицы. Доказывается, что функция $n(1, \mathbf{T}(\cdot))$ при нулевом значении полного квазиимпульса $K$ имеет в интервале $(b, 0)$ бесконечное число точек разрыва, т.е. доказано существование бесконечного числа собственных значений $H(\mathbf{0})$ на лакуне существенного спектра.

$\mathrm{B}$ настоящей работе через $C, C^{\prime}, C^{\prime \prime}$ обозначены положительные постоянные, которые могут иметь разные значения, через $\mathbf{A}, \mathbf{B}, \ldots$ - операторы, заданные некоторыми операторными матрицами; также $p^{2}=p_{1}^{2}+p_{2}^{2}+p_{3}^{2}, p q=p_{1} q_{1}+p_{2} q_{2}+p_{3} q_{3}$, $p, q \in \mathbb{T}^{3}$.

\section{2. ОПИСАНИЕ ТРЕХЧАСТИЧНОГО ОПЕРАТОРА И ФОРМУЛИРОВКА ОСНОВНЫХ РЕЗУЛЬТАТОВ}

Пусть $\mathbb{Z}^{3}$ - трехмерная целочисленная решетка и $\ell_{2}\left(\left(\mathbb{Z}^{3}\right)^{3}\right)$ - гильбертово пространство квадратично-суммируемых функций, определенных на $\left(\mathbb{Z}^{3}\right)^{3}$. Свободный гамильтониан $\widehat{H}_{0}$ системы трех произвольных квантовых частиц на решетке $\mathbb{Z}^{3}$ определяется как ограниченный самосопряженный оператор в гильбертовом пространстве $\ell_{2}\left(\left(\mathbb{Z}^{3}\right)^{3}\right)$ :

$$
\widehat{H}_{0}=\frac{1}{2 m_{1}} \Delta_{x_{1}}+\frac{1}{2 m_{2}} \Delta_{x_{2}}+\frac{1}{2 m_{3}} \Delta_{x_{3}}
$$

где $\Delta_{x_{1}}=\Delta \otimes I \otimes I, \Delta_{x_{2}}=I \otimes \Delta \otimes I$ и $\Delta_{x_{3}}=I \otimes I \otimes \Delta ; m_{\alpha}>0$ - масса частицы, $\alpha=1,2,3 ; \Delta$ - решетчатый лапласиан, который является разностным оператором, описывающим перенос частицы с узла на соседний узел, т.е.

$$
(\Delta \hat{\psi})(x)=\sum_{|s|=1}[\hat{\psi}(x)-\hat{\psi}(x+s)], \quad \hat{\psi} \in \ell_{2}\left(\mathbb{Z}^{3}\right) .
$$

Трехчастичный гамильтониан $\widehat{H}$ системы трех квантовых частиц с парными контактными потенциалами определяется как ограниченное возмущение свободного гамильтониана $\widehat{H}_{0}$ :

$$
\widehat{H}=\widehat{H}_{0}-\mu_{1} \widehat{V}_{1}-\mu_{2} \widehat{V}_{2}-\mu_{3} \widehat{V}_{3},
$$

где

$$
\left(\widehat{V}_{\alpha} \psi\right)\left(n_{1}, n_{2}, n_{3}\right)=\delta_{n_{\beta} n_{\gamma}} \psi\left(n_{1}, n_{2}, n_{3}\right), \quad\{\alpha, \beta, \gamma\}=\{1,2,3\}
$$

$\mu_{\alpha}>0$ - энергия взаимодействия двух частиц, $\delta_{n m}-$ символ Кронекера.

Пусть $L_{2}\left(\left(\mathbb{T}^{3}\right)^{n}\right), n=1,2,3$, - гильбертово пространство квадратично-интегрируемых функций, определенных на $\left(\mathbb{T}^{3}\right)^{n}, \mathbb{T}=(-\pi, \pi]$. 
С помощью преобразования Фурье и разложения в прямой операторный интеграл [9], [10] изучение спектральных свойств оператора $\widehat{H}$ сводится к исследованию спектральных свойств семейства самосопряженных ограниченных операторов $H(K), K \in \mathbb{T}^{3}$, действующих в гильбертовом пространстве $L_{2}\left(\left(\mathbb{T}^{3}\right)^{2}\right)$ по формуле

$$
H(K)=H_{0}(K)-V, \quad V=\mu_{1} V_{1}+\mu_{2} V_{2}+\mu_{3} V_{3},
$$

где $H_{0}(K)$ - оператор умножения на функцию

$$
\mathcal{E}_{K}(p, q)=\frac{1}{m_{1}} \varepsilon(p)+\frac{1}{m_{2}} \varepsilon(q)+\frac{1}{m_{3}} \varepsilon(K-p-q), \quad \varepsilon(p)=\sum_{i=1}^{3}\left(1-\cos p^{(i)}\right),
$$

и

$$
\begin{aligned}
& \left(V_{1} f\right)(p, q)=\frac{1}{(2 \pi)^{3}} \int_{\mathbb{T}^{3}} f(p, s) d s, \quad\left(V_{2} f\right)(p, q)=\frac{1}{(2 \pi)^{3}} \int_{\mathbb{T}^{3}} f(s, q) d s, \\
& \left(V_{3} f\right)(p, q)=\frac{1}{(2 \pi)^{3}} \int_{\mathbb{T}^{3}} f(s, p+q-s) d s, \quad f \in L_{2}\left(\left(\mathbb{T}^{3}\right)^{2}\right) .
\end{aligned}
$$

Введем оператор Шредингера $h_{\alpha}(k), k \in \mathbb{T}^{3}$, соответствующий двухчастичному гамильтониану на решетке $[10]$, действующий в $L_{2}\left(\mathbb{T}^{3}\right)$ по формуле

$$
h_{\alpha}(k)=h_{0}^{\alpha}(k)-\mu_{\alpha} v, \quad \alpha=1,2,3,
$$

где $h_{0}^{\alpha}(k)$ - оператор умножения на функцию $\left(1 / m_{\beta}\right) \varepsilon(p)+\left(1 / m_{\gamma}\right) \varepsilon(k-p)$ и

$$
(v f)(p)=\frac{1}{(2 \pi)^{3}} \int_{\mathbb{T}^{3}} f(s) d s .
$$

Легко проверить [10], что число $z \in \mathbb{C} \backslash \sigma_{\mathrm{ess}}\left(h_{\alpha}(k)\right)$ является собственным значением оператора $h_{\alpha}(k)$ тогда и только тогда, когда $\Delta_{\mu_{\alpha}}(k ; z)=0$, где

$$
\Delta_{\mu_{\alpha}}(k ; z)=1-\frac{\mu_{\alpha}}{(2 \pi)^{3}} \int_{\mathbb{T}^{3}} \frac{d q}{\left(1 / m_{\beta}\right) \varepsilon(q)+\left(1 / m_{\gamma}\right) \varepsilon(k-q)-z} .
$$

Пусть $C\left(\mathbb{T}^{3}\right)$ - пространство непрерывных функций, определенных на $\mathbb{T}^{3}$.

ОПРЕДЕЛЕНИЕ. Говорят, что оператор $h_{\alpha}(\mathbf{0}), \mathbf{0}=(0,0,0) \in \mathbb{T}^{3}$, имеет резонанс (виртуальный уровень) в нуле, если уравнение

$$
\varphi(p)=\frac{\mu_{\alpha}}{(2 \pi)^{3}} \frac{m_{\beta} m_{\gamma}}{m_{\beta}+m_{\gamma}} \int_{\mathbb{T}^{3}} \frac{\varphi(s) d s}{\varepsilon(s)}
$$

имеет единственное нетривиальное решение $\psi_{\alpha} \in C\left(\mathbb{T}^{3}\right)$, удовлетворяющее условию $\psi_{\alpha}(\mathbf{0}) \neq 0$ (см. также [4], [10]).

Простое вычисление показывает, что оператор $h_{\alpha}(\mathbf{0})$ имеет резонанс в нуле тогда и только тогда, когда $\mu_{\alpha}=\mu_{\alpha}^{0}$, где

$$
\mu_{\alpha}^{0}=8 \pi^{3} \frac{m_{\beta}+m_{\gamma}}{m_{\beta} m_{\gamma}}\left(\int_{\mathbb{T}^{3}} \frac{d s}{\varepsilon(s)}\right)^{-1} .
$$


Если уравнение (2.1) не имеет решения, то $\lambda=0$ не является собственным значением оператора $h_{\alpha}(\mathbf{0})$. В этом случае нуль называется регулярной точкой оператора $h_{\alpha}(\mathbf{0})$ (см. [12]).

Оператор $H_{\alpha}(K)=H_{0}(K)-V_{\alpha}$ называется канальным оператором и представляется в виде прямого интеграла [9], [10]:

$$
H_{\alpha}(K)=\int_{\mathbb{T}^{3}} \oplus\left[\tilde{h}_{\alpha}(K-p)+\frac{1}{m_{\alpha}} \varepsilon(p)\right] d p,
$$

где оператор $\tilde{h}_{\alpha}(p)$ унитарно эквивалентен оператору $h_{\alpha}(p)$.

Отсюда и из теоремы о спектре тензорных произведений операторов имеем

$$
\sigma\left(H_{\alpha}(K)\right)=\bigcup_{p \in \mathbb{T}^{3}}\left[\sigma\left(h_{\alpha}(K-p)\right) \cup\left\{\frac{1}{m_{\alpha}} \varepsilon(p)\right\}\right]
$$

Из теоремы Вейля о существенном спектре [13] следует, что существенный спектр $\sigma_{\text {ess }}\left(h_{\alpha}(k)\right)$ оператора $h_{\alpha}(k)$ не меняется при компактном возмущении $\mu_{\alpha} v$ и совпадает со спектром невозмущенного оператора $h_{0}^{\alpha}(k)$. При этом $\sigma_{\mathrm{ess}}\left(h_{\alpha}(k)\right)$ совпадает с областью значения функции $\left(1 / m_{\beta}\right) \varepsilon(\cdot)+\left(1 / m_{\gamma}\right) \varepsilon(k-\cdot)$, т.е.

$$
\sigma_{\mathrm{ess}}\left(h_{\alpha}(k)\right)=\sigma\left(h_{0}^{\alpha}(k)\right)=\left[\varepsilon_{\min }^{(\alpha)}(k), \varepsilon_{\max }^{(\alpha)}(k)\right],
$$

где

$$
\varepsilon_{\min }^{(\alpha)}(k)=\min _{p}\left[\frac{1}{m_{\beta}} \varepsilon(p)+\frac{1}{m_{\gamma}} \varepsilon(k-p)\right], \quad \varepsilon_{\max }^{(\alpha)}(k)=\max _{p}\left[\frac{1}{m_{\beta}} \varepsilon(p)+\frac{1}{m_{\gamma}} \varepsilon(k-p)\right] .
$$

Поэтому в силу (2.2) имеем

$$
\sigma\left(H_{\alpha}(K)\right)=\bigcup_{p \in \mathbb{T}^{3}}\left(\left[\varepsilon_{\min }^{(\alpha)}(K-p)+\frac{1}{m_{\alpha}} \varepsilon(p), \varepsilon_{\max }^{(\alpha)}(K-p)+\frac{1}{m_{\alpha}} \varepsilon(p)\right] \cup \sigma_{\operatorname{disc}}\left(h_{\alpha}(K-p)\right)\right)
$$

или

$$
\sigma\left(H_{\alpha}(K)\right)=\left[m_{K}, M_{K}\right] \cup \bigcup_{p \in \mathbb{T}^{3}} \sigma_{\text {disc }}\left(h_{\alpha}(K-p)\right),
$$

где $m_{K}=\min _{p, q} \mathcal{E}_{K}(p, q), M_{K}=\max _{p, q} \mathcal{E}_{K}(p, q)$.

Следующая лемма доказана в работе [10].

Лемма 1. Для любого $K \in \mathbb{T}^{3}$ существенный спектр $\sigma_{\mathrm{ess}}(H(K))$ оператора $H(K)$ состоит из обгединения спектров операторов каналов $H_{\alpha}(K)$, m.e.

$$
\sigma_{\text {ess }}(H(K))=\sigma\left(H_{1}(K)\right) \cup \sigma\left(H_{2}(K)\right) \cup \sigma\left(H_{3}(K)\right) .
$$

Теперь сформулируем основные результаты настоящей работы. 
Пусть $W_{\alpha}(z)$ - оператор умножения на функцию $\left[\Delta_{\mu_{\alpha}}\left(K-p_{\alpha} ; z-\left(1 / m_{i}\right) \varepsilon\left(p_{\alpha}\right)\right)\right]^{-1}$, $\alpha=1,2,3$, где $p_{1}=p, p_{2}=q, p_{3}=K-p, p, q \in \mathbb{T}^{3}$. Из монотонности функции $\Delta_{\mu_{\alpha}}(k ; z)$ по $z$ и неравенства $\Delta_{\mu_{\alpha}}(k ; z)>0$ при $z<\inf \sigma\left(h_{\alpha}(k)\right)$ следует неравенство $\Delta_{\mu_{\alpha}}\left(p ; z-\left(1 / m_{\alpha}\right) \varepsilon(p)\right)>0$ для всех $z<\inf \sigma\left(h_{\alpha}(p)\right)$ и $p \in \mathbb{T}^{3}$. Отсюда и из равенства (2.3) следует, что $W_{\alpha}(z)$ является положительным оператором при всех $z<\inf \sigma_{\text {ess }}\left(H_{\alpha}(K)\right)$.

Определим оператор $\mathbf{T}(z), z<\inf \left\{\sigma\left(H_{1}(K)\right) \cup \sigma\left(H_{2}(K)\right)\right\}, z \notin \sigma\left(H_{3}(K)\right)$, в гильбертовом пространстве $L_{2}^{(2)}\left(\mathbb{T}^{3}\right)$, заданный матрицей

$$
\mathbf{T}(z)=\left(\begin{array}{cc}
0 & W_{1}^{1 / 2}(z) K_{12}(z) W_{2}^{1 / 2}(z) \\
W_{2}^{1 / 2}(z) K_{21}(z) W_{1}^{1 / 2}(z) & 0
\end{array}\right)+\left(\begin{array}{ll}
T_{11}(z) & T_{12}(z) \\
T_{21}(z) & T_{22}(z)
\end{array}\right)
$$

где $T_{i j}(z)=W_{i}^{1 / 2}(z) K_{i 3}(z) W_{3}(z) K_{3 j}(z) W_{j}^{1 / 2}(z), K_{i j}(z)$ - интегральный оператор, порожденный ядром $K_{i j}\left(z ; p, p^{\prime}\right)$, такой, что $K_{i j}(z)=\left[K_{j i}(z)\right]^{*}$,

$$
\begin{gathered}
K_{12}\left(z ; p, p^{\prime}\right)=\frac{(2 \pi)^{-3} \sqrt{\mu_{1} \mu_{2}}}{\mathcal{E}_{K}\left(p, p^{\prime}\right)-z}, \quad K_{13}\left(z ; p, p^{\prime}\right)=\frac{(2 \pi)^{-3} \sqrt{\mu_{1} \mu_{3}}}{\mathcal{E}_{K}\left(p, p^{\prime}-p\right)-z} \\
K_{23}\left(z ; p, p^{\prime}\right)=\frac{(2 \pi)^{-3} \sqrt{\mu_{2} \mu_{3}}}{\mathcal{E}_{K}\left(p^{\prime}-p, p\right)-z}
\end{gathered}
$$

Пусть $n(\lambda, A)$ - число собственных значений (с учетом кратности) самосопряженного оператора $A$, лежащих правее $\lambda, \lambda>\sup \sigma_{\mathrm{ess}}(A)$.

ЛЕмма 2. Число $z_{0}<\inf \sigma\left(H_{1}(K)\right) \cup \sigma\left(H_{2}(K)\right)$ при $z_{0} \notin \sigma\left(H_{3}(K)\right)$ является собственным значением оператора $H(K)$ тогда и только тогда, когда функиия $n(1, \mathbf{T}(z))$ имеет разрьв в точке $z=z_{0}$.

Пусть $m$ - положительное число. Положим

$$
\mu_{3}^{*}=(2 \pi)^{3}\left[\min _{k} \int_{\mathbb{T}^{3}} \frac{d q}{\left(1 / m_{1}\right) \varepsilon(q)+\left(1 / m_{2}\right) \varepsilon(k-q)+m}\right]^{-1} .
$$

Tеорема 1. 1. Пусть $\mu_{\alpha} \leqslant \mu_{\alpha}^{0}, \alpha=1,2, \mu_{3}>\mu_{3}^{*}$. Тогда существенный спектр $\sigma_{\mathrm{ess}}(H(\mathbf{0}))$ содержит лакуны, точнее $\sigma_{\mathrm{ess}}(H(\mathbf{0}))=\left[a_{3}, b_{3}\right] \cup\left[0, M_{\mathbf{0}}\right], a_{3}<b_{3}<-m$.

2. Пусть $\mu_{1}=\mu_{1}^{0}, \mu_{2}=\mu_{2}^{0}, \mu_{3}>\mu_{3}^{*}$. Тогда оператор $H(\mathbf{0})$ в лакуне существенного спектра $\left(b_{3}, 0\right)$ имеет бесконечное число собственных значений.

Приведем схему доказательства этой теоремы. Для доказательства утверждения 1 теоремы 1 используется лемма 1. В разделах 3 и 4 доказывается лемма 2, которая играет важную роль при доказательстве утверждения 2 теоремы 1. В разделе 5 с помощью лемм 9-13 показано, что функция $n(1, \mathbf{T}(\cdot))$ имеет бесконечное число точек разрыва в $\left(b_{3} / 2,0\right)$, при этом из леммы 2 следует доказательство утверждения 2 теоремы 1. 


\section{3. УРАВНЕНИЕ ФАДДЕЕВА ДЛЯ ТРЕХЧАСТИЧНОГО ОПЕРАТОРА ШРЕДИНГЕРА}

При каждом $z \notin \sigma\left(H_{\mathrm{ess}}(K)\right)$ определим операторы $\mathbf{B}(z)$ и $\mathbf{A}(z)$ соответственно в гильбертовых пространствах $L_{2}^{(2)}\left(\mathbb{T}^{3}\right)$ и $L_{2}^{(3)}\left(\mathbb{T}^{3}\right)$, заданные матрицами

$$
\begin{aligned}
\mathbf{B}(z)= & \left(\begin{array}{ccc}
0 & W_{1}(z) K_{12}(z) \\
W_{2}(z) K_{21}(z) & 0
\end{array}\right)+ \\
& +\left(\begin{array}{lll}
W_{1}(z) K_{13}(z) W_{3}(z) K_{31}(z) & W_{1}(z) K_{13}(z) W_{3}(z) K_{32}(z) \\
W_{2}(z) K_{23}(z) W_{3}(z) K_{31}(z) & W_{2}(z) K_{23}(z) W_{3}(z) K_{32}(z)
\end{array}\right) \\
& \mathbf{A}(z)=\left(\begin{array}{ccc}
0 & W_{1}(z) K_{12}(z) & W_{1}(z) K_{13}(z) \\
W_{2}(z) K_{21}(z) & 0 & W_{2}(z) K_{23}(z) \\
W_{3}(z) K_{31}(z) & W_{3}(z) K_{32}(z) & 0
\end{array}\right)
\end{aligned}
$$

Лемма 3. Число $z \in \mathbb{C} \backslash \sigma_{\mathrm{ess}}(H(K))$, где $\mathbb{C}-$ комплексная плоскость, является собственным значением оператора $H(K)$ тогда и только тогда, когда компактныи оператор $\mathbf{B}(z)$ имеет собственное значение, равное единище, причем кратности чисел z и 1 совпадают.

ДоказАтеЛьство. Пусть $z \in \mathbb{C} \backslash \sigma_{\text {ess }}(H(K))$ - собственное значение оператора $H(K), f$ - соответствующий собственный вектор, т.е. уравнение $H(K) f=z f$ имеет нетривиальное решение $f$. Поскольку при $z \in \mathbb{C} \backslash \sigma_{\mathrm{ess}}(H(K))$ оператор $R_{0}(z)$ ограничен в $L_{2}\left(\left(\mathbb{T}^{3}\right)^{2}\right)$, имеем

$$
f=R_{0}(z) V f
$$

Отсюда следует, что число $z$ является собственным значением оператора $H(K)$ тогда и только тогда, когда единица является собственным значением $R_{0}(z) V$, причем кратности чисел $z$ и 1 совпадают. Положим

$$
\varphi_{\alpha}=\sqrt{\mu_{\alpha}} V_{\alpha} f, \quad \alpha=1,2,3
$$

Из равенства (3.1) имеем

$$
f(p, q)=\frac{1}{\mathcal{E}_{K}(p, q)-z}\left[\sqrt{\mu_{1}} \varphi_{1}(p)+\sqrt{\mu_{2}} \varphi_{2}(q)+\sqrt{\mu_{3}} \varphi_{3}(p+q)\right] .
$$

Подставляя выражение (3.3) в (3.2), получаем, что функции $\varphi_{\alpha}, \alpha=1,2,3$, удовлетворяют системе интегральных уравнений

$$
\begin{aligned}
\left(W_{\alpha}^{-1}(z) \varphi_{1}\right)(p)= & \frac{\sqrt{\mu_{1} \mu_{2}}}{(2 \pi)^{3}} \int \frac{\varphi_{2}\left(p^{\prime}\right) d p^{\prime}}{\mathcal{E}_{K}\left(p, p^{\prime}\right)-z}+\frac{\sqrt{\mu_{1} \mu_{3}}}{(2 \pi)^{3}} \int \frac{\varphi_{3}\left(p+p^{\prime}\right) d p^{\prime}}{\mathcal{E}_{K}\left(p, p^{\prime}\right)-z}, \\
\left(W_{\alpha}^{-1}(z) \varphi_{2}\right)(q)= & \frac{\sqrt{\mu_{2} \mu_{1}}}{(2 \pi)^{3}} \int \frac{\varphi_{1}\left(p^{\prime}\right) d p^{\prime}}{\mathcal{E}_{K}\left(p^{\prime}, q\right)-z}+\frac{\sqrt{\mu_{2} \mu_{3}}}{(2 \pi)^{3}} \int \frac{\varphi_{3}\left(p^{\prime}+q\right) d p^{\prime}}{\mathcal{E}_{K}\left(p^{\prime}, q\right)-z}, \\
\left(W_{\alpha}^{-1}(z) \varphi_{3}\right)(p+q)= & \frac{\sqrt{\mu_{3} \mu_{1}}}{(2 \pi)^{3}} \int \frac{\varphi_{1}\left(p^{\prime}\right) d p^{\prime}}{\mathcal{E}_{K}\left(p^{\prime}, p+q-p^{\prime}\right)-z}+ \\
& +\frac{\sqrt{\mu_{3} \mu_{2}}}{(2 \pi)^{3}} \int \frac{\varphi_{2}\left(p+q-p^{\prime}\right) d p^{\prime}}{\mathcal{E}_{K}\left(p^{\prime}, p+q-p^{\prime}\right)-z} .
\end{aligned}
$$


Пользуясь периодичностью подынтегральных функций в этой системе уравнений, производя замену переменных $p+p^{\prime}=s, p^{\prime}+q=s$ и $p+q-p^{\prime}=s^{\prime}$ во вторых подынтегральных выражениях и учитывая, что $\Delta_{\mu_{\alpha}}(z ; p) \neq 0, \alpha=1,2,3$, при $z \in$ $\mathbb{C} \backslash \sigma_{\mathrm{ess}}(H(K)), p \in \mathbb{T}^{3}$, убеждаемся, что $\varphi_{\alpha}, \alpha=1,2,3$, удовлетворяют системе интегральных уравнений

$$
\begin{aligned}
& \varphi_{1}=W_{1}(z) K_{12}(z) \varphi_{2}+W_{1}(z) K_{13}(z) \varphi_{3}, \\
& \varphi_{2}=W_{2}(z) K_{21}(z) \varphi_{1}+W_{2}(z) K_{23}(z) \varphi_{3}, \\
& \varphi_{3}=W_{3}(z) K_{31}(z) \varphi_{1}+W_{3}(z) K_{32}(z) \varphi_{2},
\end{aligned}
$$

или

$$
\Phi=\mathbf{A}(z) \Phi, \quad \Phi=\left(\varphi_{1}, \varphi_{2}, \varphi_{3}\right) .
$$

При этом вектор $f$, определенный по формуле (3.3), является собственным вектором оператора $R_{0}(z) V$, соответствующим собственному значению 1 , тогда и только тогда, когда $\Phi=\left(\varphi_{1}, \varphi_{2}, \varphi_{3}\right)$ является собственным вектором оператора $\mathbf{A}(z)$, соответствующим собственному значению 1. Количества таких линейно независимых собственных векторов $f$ и $\Phi$ совпадают. Поэтому кратности собственного значения 1 операторов $\mathbf{A}(z)$ и $R_{0}(z) V$ совпадают.

Подставляя выражение для $\varphi_{3}$ в первое и второе равенства в $(3.4)$, получаем

$$
\begin{aligned}
& \varphi_{1}=W_{1}(z) K_{12}(z) \varphi_{2}+W_{1}(z) K_{13}(z)\left(W_{3}(z) K_{31}(z) \varphi_{1}+W_{3}(z) K_{32}(z) \varphi_{2}\right), \\
& \varphi_{2}=W_{2}(z) K_{21}(z) \varphi_{1}+W_{2}(z) K_{23}(z)\left(W_{3}(z) K_{31}(z) \varphi_{1}+W_{3}(z) K_{32}(z) \varphi_{2}\right),
\end{aligned}
$$

или

$$
\mathbf{B}(z) \Psi=\Psi, \quad \Psi=\left(\varphi_{1}, \varphi_{2}\right),
$$

т.е. число 1 является собственным значением оператора $\mathbf{B}(z)$.

И наоборот, если 1 - собственное значение, а $\Psi=\left(\varphi_{1}, \varphi_{2}\right)$ - соответствующая собственная функция оператора $\mathbf{B}(z)$, то число 1 является собственным значением оператора $\mathbf{A}(z)$, соответствующим собственному вектору $\Phi=\left(\varphi_{1}, \varphi_{2}, \varphi_{3}\right)$, где $\varphi_{3}=W_{3}(z) K_{31}(z) \varphi_{1}+W_{3}(z) K_{32}(z) \varphi_{2}$. Легко проверить, что количества линейно независимых решений уравнений (3.4) и (3.5) совпадают. Следовательно, кратности собственного значения 1 операторов $\mathbf{A}(z)$ и $\mathbf{B}(z)$ совпадают.

Ядро интегрального оператора $K_{i j}(z)$ при каждом $z \notin \sigma\left(H_{0}(K)\right)$ является непрерывной функцией в $\left(\mathbb{T}^{3}\right)^{2}$, поэтому $K_{i j}(z)$ принадлежит классу операторов со следом. Отсюда и из ограниченности оператора $W_{i}(z), i=1,2,3$, вытекает, что каждый элемент матричных операторов $\mathbf{A}(z)$ и $\mathbf{B}(z)$ принадлежит классу операторов со следом. Следовательно, операторы $\mathbf{A}(z)$ и $\mathbf{B}(z)$ принадлежат классу операторов со следом.

ЗАмЕчание. Уравнение $\mathbf{A}(z) \Phi=\Phi$ есть аналог уравнения Фаддеева, которое получено для трехчастичного непрерывного оператора Шредингера [3].

Оператор-функция $\mathbf{B}(z)$ является аналитической при $z \in \mathbb{C} \backslash \sigma_{\mathrm{ess}}(H(K))$ и $\mathbf{B}(z)$ является оператором со следом, поэтому детерминант $D(\lambda, z)$ оператора $\mathbf{E}-\lambda^{-1} \mathbf{B}(z)$ определен и аналитичен при $z \in \mathbb{C} \backslash \sigma_{\mathrm{ess}}(H(K))$ и $\lambda \neq 0$, где $\mathbf{E}$ - единичный оператор, действующий в $L_{2}^{(2)}\left(\mathbb{T}^{3}\right)$. Из теоремы XIII.105 в монографии [13] вытекает 
ЛЕмма 4. Число $\lambda \neq 0$ является собственным значением оператора $\mathbf{B}(z), z \in$ $\mathbb{C} \backslash \sigma_{\mathrm{ess}}(H(K))$, тогда и только тогда, когда $D(\lambda, z)=0$.

Из лемм 3 и 4 вытекает

Лемма 5. Число $z \in \mathbb{C} \backslash \sigma_{\mathrm{ess}}(H(K))$ является собственным значением оператора $H(K)$ тогда и только тогда, когда $D(1, z)=0$.

\section{4. ПРИНЦИП БИРМАНА-ШВИНГЕРА}

В этом разделе докажем лемму 2, необходимую для доказательства основного результата.

ЛЕмма 6. Каждое ненулевое собственное значение оператора $\mathbf{B}(z)$ при $z<$ $\inf \left\{\sigma_{1}(H(K)) \cup \sigma_{2}(H(K))\right\}, z \notin \sigma_{3}(H(K))$, является собственным значением оператора $\mathbf{T}(z)$ с той же кратностью. При этом число $z<\inf \left\{\sigma_{1}(H(K)) \cup \sigma_{2}(H(K))\right\}$, $z \notin \sigma_{3}(H(K))$, является собственным значением оператора $H(K)$ тогда и только тогда, когда оператор $\mathbf{T}(z)$ имеет собственное значение, равное единице, причем кратности собственных значений z и 1 совпадают (приниип Бирмана-Швингера).

ДоказАтельство. Из неравенства $z<\inf \left\{\sigma_{1}(H(K)) \cup \sigma_{2}(H(K))\right\}$ следует, что $W_{\alpha}(z)>0, \alpha=1,2$. Представим оператор $\mathbf{B}(z)$ в виде

$$
\mathbf{B}(z)=\mathbf{W}^{1 / 2}(z)\left[\mathbf{B}_{0}(z)+\mathbf{B}_{1}(z)\right]
$$

где

$$
\begin{gathered}
\mathbf{W}^{1 / 2}(z)=\left(\begin{array}{cc}
W_{1}^{1 / 2}(z) & 0 \\
0 & W_{2}^{1 / 2}(z)
\end{array}\right), \quad \mathbf{B}_{0}(z)=\left(\begin{array}{ccc}
0 & W_{1}^{1 / 2}(z) K_{12}(z) \\
W_{2}^{1 / 2}(z) K_{21}(z) & 0
\end{array}\right), \\
\mathbf{B}_{1}(z)=\left(\begin{array}{ll}
W_{1}^{1 / 2}(z) K_{13}(z) W_{3}(z) K_{31}(z) & W_{1}^{1 / 2}(z) K_{13}(z) W_{3}(z) K_{32}(z) \\
W_{2}^{1 / 2}(z) K_{23}(z) W_{3}(z) K_{31}(z) & W_{2}^{1 / 2}(z) K_{23}(z) W_{3}(z) K_{32}(z)
\end{array}\right) .
\end{gathered}
$$

Поскольку для ограниченных операторов $A$ и $B$ каждое ненулевое собственное значение оператора $A B$ является собственным значением $B A$ с той же кратностью (см. [14]), собственное значение оператора $\mathbf{B}(z)$, равное единице, является собственным значением оператора $\left[\mathbf{B}_{0}(z)+\mathbf{B}_{1}(z)\right] \mathbf{W}^{1 / 2}(z)=\mathbf{T}(z)$ с той же кратностью.

Теорема 2. Число $z_{0}<\inf \left\{\sigma_{1}(H(K)) \cup \sigma_{2}(H(K))\right\}, z_{0} \notin \sigma_{3}(H(K))$, является регулярной точкой оператора $H(K)$, если и только если функиия $n(1, \mathbf{T}(z))$ является непрерьвной в точке $z=z_{0}$.

ДокАзАТЕЛЬство. Необходимость. Предположим, что $z=z_{0}$ - регулярная точка оператора $H(K)$. Тогда из леммы 6 вытекает, что оператор $\mathbf{E}-\mathbf{T}\left(z_{0}\right)$ обратим. Из непрерывности оператор-функции $\lambda^{-1} \mathbf{T}(z)$ по $(\lambda, z)$ в $(0, \infty) \times \mathbb{R} \backslash \sigma_{\mathrm{ess}}(H(K))$ и компактности $\mathbf{T}(z)$ вытекает, что оператор $\mathbf{E}-\lambda^{-1} \mathbf{T}(z)$ является обратимым при всех $(\lambda, z)$, лежащих в некоторой окрестности точки $\left(1, z_{0}\right)$. Поэтому для некоторого $\rho>0$ получаем $\sigma(\mathbf{T}(z)) \cap(1-\rho, 1+\rho)=\varnothing$ при всех $z \in\left[z_{0}-\rho, z_{0}+\rho\right]$. Отсюда по определению функции $n(a, \mathbf{T}(z))$ для всех $\xi$ и $\delta \in\left[0, z_{0}+\rho\right)$ имеем

$$
n\left(1 \pm \delta, \mathbf{T}\left(z_{0} \pm \xi\right)\right)=n\left(1, \mathbf{T}\left(z_{0} \pm \xi\right)\right) .
$$


Далее, пользуясь неравенством Вейля $n(a+b, A+B) \leqslant n(a, A)+n(b, B)$ для компактных операторов $A, B[15]$, для некоторого $\eta_{0} \in(0, \delta)$ и всех малых $\xi>0$ получаем

$$
n\left(1+\eta_{0}, \mathbf{T}\left(z_{0} \pm \xi\right)\right) \leqslant n\left(1, \mathbf{T}\left(z_{0}\right)\right)+n\left(\eta_{0}, \mathbf{T}\left(z_{0} \pm \xi\right)-\mathbf{T}\left(z_{0}\right)\right)=n\left(1, \mathbf{T}\left(z_{0}\right)\right) .
$$

Рассуждая аналогично, при малых $\xi>0$ получаем обратное неравенство

$$
n\left(1, \mathbf{T}\left(z_{0}\right)\right)=n\left(1+\eta_{0}, \mathbf{T}\left(z_{0}\right)\right) \leqslant n\left(1, \mathbf{T}\left(z_{0} \pm \xi\right)\right) .
$$

Поэтому для всех малых $\xi>0$ имеет место равенство $n\left(1, \mathbf{T}\left(z_{0} \pm \xi\right)\right)=n\left(1, \mathbf{T}\left(z_{0}\right)\right)$, т.е. функция $n(1, \mathbf{T}(z))$ непрерывна в точке $z=z_{0}$.

Достаточность. Предположим обратное. Пусть функция $n(1, \mathbf{T}(z))$ непрерывна в точке $z_{0}<\inf \left\{\sigma\left(H_{1}(K)\right) \cup \sigma\left(H_{2}(K)\right)\right\}, z_{0} \notin \sigma_{3}(H(K)), z_{0}$ - дискретное собственное значение оператора $H(K)$.

Рассуждая как и выше и используя неравенство Вейля, получаем, что для некоторого $\delta_{0}>0, c_{0}=c_{0}\left(\delta_{0}\right)>0$ и для всех $\varepsilon \in\left[-c_{0}, c_{0}\right]$ выполняются равенства

$$
n\left(1, \mathbf{T}\left(z_{0}\right)\right)=n\left(1+\delta_{0}, \mathbf{T}\left(z_{0}\right)\right)=n\left(1+\delta_{0} / 4, \mathbf{T}\left(z_{0}+\varepsilon\right)\right) .
$$

В силу леммы $5 D\left(1, z_{0}\right)=0$. Пусть $\Gamma_{\delta}$ - граница комплексной $\delta$-окрестности $U_{\delta}\left(z_{0}\right)$ точки $z_{0}$. При этом в силу малости $\delta$ для всех $z \in \Gamma_{\delta}$ имеет место $D(1, z) \neq 0$. Положим

$$
d=\min _{z \in \Gamma_{\delta}}|D(1, z)|, \quad \psi_{\varepsilon}(z)=D(1+\varepsilon, z)-D(1, z) .
$$

Поскольку функция $D(\lambda, z)$ является непрерывной, существует число $\rho=\rho(\delta)>0$ такое, что для всех $\varepsilon \in[-\rho, \rho]$ и $z \in \Gamma_{\delta}$ выполняется неравенство $\left|\psi_{\varepsilon}(z)\right|<d$. Таким образом, для каждого фиксированного $\varepsilon \in[-\rho, \rho]$ функции $D(1, z)$ и $\psi_{\varepsilon}(z)$, определенные на $\overline{U_{\delta}\left(z_{0}\right)}$, удовлетворяют условиям теоремы Руше. Поэтому количества нулей функций $D(1, z)$ и $D(1+\varepsilon, z)$, лежащих в $U_{\delta}\left(z_{0}\right)$, совпадают. Пусть $D\left(1+\varepsilon, z_{\varepsilon}\right)=0, \varepsilon>0$. В силу леммы 4 число $1+\varepsilon$ есть собственное значение оператора $\mathbf{B}\left(z_{\varepsilon}\right)$, поэтому с учетом предложения (см. ниже) $z_{\varepsilon}$ является вещественным. В силу леммы 6 для всех $\varepsilon \in\left(0, \rho^{\prime}\right)$ число $1+\varepsilon$ является собственным значением оператора $\mathbf{T}\left(z_{\varepsilon}\right)$. Отсюда и из равенства (4.1) для всех $\varepsilon \in\left(0, \rho^{\prime}\right)$ имеем

$$
\begin{aligned}
n\left(1, \mathbf{T}\left(z_{\varepsilon}\right)\right)-n\left(1, \mathbf{T}\left(z_{0}\right)\right) & \geqslant n\left(1+\frac{\varepsilon}{2}, \mathbf{T}\left(z_{\varepsilon}\right)\right)-n\left(1, \mathbf{T}\left(z_{0}\right)\right) \geqslant \\
& \geqslant 1+n\left(1+\frac{\delta_{0}}{4}, \mathbf{T}\left(z_{\varepsilon}\right)\right)-n\left(1, \mathbf{T}\left(z_{0}\right)\right)=1 .
\end{aligned}
$$

Следовательно, $n\left(1, \mathbf{T}\left(z_{0}\right)\right) \neq \lim _{\varepsilon \rightarrow 0} n\left(1, \mathbf{T}\left(z_{\varepsilon}\right)\right)$, т.е. функция $n(1, \mathbf{T}(z))$ не является непрерывной в точке $z=z_{0}$, что противоречит нашему предположению.

Отметим, что оператор $\mathbf{B}(z)$ определен при каждом $z \notin \sigma_{\mathrm{ess}}(H(K))$.

ПрЕДЛОЖЕНИЕ. Предположим, что число $\lambda>1$ является собственным значением оператора $\mathbf{B}(z), z \notin \sigma_{\mathrm{ess}}(H(K))$. Тогда $\operatorname{Im} z=0$.

ДокАЗАтельство. Пусть $\Psi=\left(\varphi_{1}, \varphi_{2}\right) \in L_{2}^{(2)}\left(\mathbb{T}^{3}\right)$ - собственный вектор, соответствующий собственному значению $\lambda>1$ оператора $\mathbf{B}(z), z \notin \sigma_{\mathrm{ess}}(H(K))$ :

$$
\begin{aligned}
& \lambda \varphi_{1}=W_{1}(z) K_{12}(z) \varphi_{2}+W_{1}(z) K_{13}(z)\left(W_{3}(z) K_{31}(z) \varphi_{1}+W_{3}(z) K_{32}(z) \varphi_{2}\right), \\
& \lambda \varphi_{2}=W_{2}(z) K_{21}(z) \varphi_{1}+W_{2}(z) K_{23}(z)\left(W_{3}(z) K_{31}(z) \varphi_{1}+W_{3}(z) K_{32}(z) \varphi_{2}\right) .
\end{aligned}
$$


Отсюда, полагая $\varphi_{3}=W_{3}(z) K_{31}(z) \varphi_{1}+W_{3}(z) K_{32}(z) \varphi_{2}$ и учитывая существование оператора $W_{i}^{-1}(z), i=1,2,3$, при $z \notin \sigma_{\mathrm{ess}}(H(K))$, имеем

$$
\begin{aligned}
\lambda \varphi_{1} & =\lambda K_{11}(z) \varphi_{1}+K_{12}(z) \varphi_{2}+K_{13}(z) \varphi_{3}, \\
\lambda \varphi_{2} & =K_{21}(z) \varphi_{2}+\lambda K_{22}(z) \varphi_{1}+K_{23}(z) \varphi_{3}, \\
\varphi_{3} & =K_{31}(z) \varphi_{1}+K_{32}(z) \varphi_{2}+K_{33}(z) \varphi_{3},
\end{aligned}
$$

где $K_{11}, K_{22}, K_{33}$ - операторы умножения сответственно на функции

$$
\frac{\mu_{1}}{(2 \pi)^{3}} \int \frac{d q^{\prime}}{\mathcal{E}_{K}\left(p, q^{\prime}\right)-z}, \quad \frac{\mu_{2}}{(2 \pi)^{3}} \int \frac{d p^{\prime}}{\mathcal{E}_{K}\left(p^{\prime}, q\right)-z}, \quad \frac{\mu_{3}}{(2 \pi)^{3}} \int \frac{d p^{\prime}}{\mathcal{E}_{K}\left(p^{\prime}, p-p^{\prime}\right)-z} .
$$

Равенства (4.2) запишем в операторной форме

$$
\mathbf{A}_{\lambda} \Phi=\mathbf{K}_{\lambda}(z) \Phi, \quad \Phi=\left(\varphi_{1}, \varphi_{2}, \varphi_{3}\right) .
$$

Выделяя вещественные и мнимые части функции $\left(\mathcal{E}_{K}(p, q)-z\right)^{-1}$, оператор $\mathbf{K}_{\lambda}(z)$ можно представить через самосопряженные операторы $\mathbf{L}_{\lambda}$ и $\mathbf{M}_{\lambda}$ в виде

$$
\mathbf{K}_{\lambda}(z)=\mathbf{L}_{\lambda}+i \operatorname{Im} z \mathbf{M}_{\lambda}
$$

где элементы $M_{i j}$ операторной матрицы $\mathbf{M}_{\lambda}$ действуют в пространстве $L_{2}\left(\mathbb{T}^{3}\right)$ по формулам

$$
\begin{array}{ll}
\left(M_{11} \varphi\right)(p)=\lambda \mu_{1} \int M(p, s) d s \varphi(p), & \left(M_{22} \varphi\right)(q)=\lambda \int M(s, q) d s \varphi(q), \\
\left(M_{33} \varphi\right)(p)=\mu_{3} \int M(s, p-s) d s \varphi(p), & \left(M_{12} \varphi\right)(p)=\sqrt{\mu_{1} \mu_{2}} \int M(p, s) \varphi(s) d s, \\
\left(M_{13} \varphi\right)(p)=\sqrt{\mu_{1} \mu_{3}} \int M(p, s-p) \varphi(s) d s, & \left(M_{23} \varphi\right)(q)=\sqrt{\mu_{2} \mu_{3}} \int M(s-q, q) \varphi(s) d s, \\
M_{21}=M_{12}^{*}, M_{31}=M_{13}^{*}, M_{32}=M_{23}^{*}, \text { где } &
\end{array}
$$$$
M(p, q)=\frac{1}{(2 \pi)^{3}} \frac{1}{\left[\mathcal{E}_{K}(p, q)-\operatorname{Re} z\right]^{2}+(\operatorname{Im} z)^{2}} .
$$

Умножая скалярно равенство $\mathbf{A}_{\lambda} \Phi=\mathbf{L}_{\lambda} \Phi+i \operatorname{Im} z \mathbf{M}_{\lambda} \Phi$ на вектор $\Phi$ и учитывая самосопряженность операторов $\mathbf{A}_{\lambda}, \mathbf{L}_{\lambda}$ и $\mathbf{M}_{\lambda}$, получаем

$$
\operatorname{Im} z\left(\mathbf{M}_{\lambda} \Phi, \Phi\right)=0 \text {. }
$$

Из неравенства Коши-Буняковского следует, что

$$
\left|\left(M_{i j} \varphi_{i}, \varphi_{j}\right)\right|^{2} \leqslant\left(M_{i i} \varphi_{i}, \varphi_{i}\right)\left(M_{j j} \varphi_{j}, \varphi_{j}\right)
$$

Отсюда и из соотношения $\Phi=\left(\varphi_{1}, \varphi_{2}\right) \neq(0,0)$ вытекает, что $\left(\mathbf{M}_{1} \Phi, \Phi\right)>0$. Следовательно,

$$
\begin{aligned}
\left(\mathbf{M}_{\lambda} \Phi, \Phi\right) & >\left(\mathbf{M}_{\lambda} \Phi, \Phi\right)-\left(\mathbf{M}_{1} \Phi, \Phi\right)= \\
& =(\lambda-1) \iint M(p, s)\left|\varphi_{1}(p)\right|^{2} d s+(\lambda-1) \iint M(s, q) d s\left|\varphi_{2}(q)\right|^{2} d q \geqslant 0,
\end{aligned}
$$

поэтому из равенства (4.3) имеем $\operatorname{Im} z=0$.

Доказательство леммы 2 следует из теоремы 2. 


\section{5. ДОКАЗАТЕЛЬСТВО ТЕОРЕМЫ 1}

Докажем возможность появления лакуны существенного спектра оператора $H(\mathbf{0})$.

Лемма 7. 1. Пусть $\mu_{3}>\mu_{3}^{*}$. Тогда для всех $k \in \mathbb{T}^{3}$ оператор $h_{3}(k)$ имеет единственное собственное значение, меньшее $-m$.

2. Пусть $\mu_{\alpha} \leqslant \mu_{\alpha}^{0}, \alpha=1,2$. Тогда для всех $k \in \mathbb{T}^{3}$ оператор $h_{\alpha}(k)$ не имеет собственных значений ниже нуля.

ДокАзАТЕЛЬСтво. 1. Покажем, что функция $\Delta_{\mu_{3}}(k ; \cdot)$ имеет единственный нуль, меньший $-m$. Из монотонности функции $\Delta_{\mu_{3}}(k ; z)$ по $z$ и $\mu_{3}$ для всех $z \in(-m, 0)$ получаем

$$
\Delta_{\mu_{3}}(k ; z) \leqslant \Delta_{\mu_{3}}(k ;-m) \leqslant \Delta_{\mu_{3}^{*}}(k ;-m) \leqslant 0 .
$$

В силу $\lim _{z \rightarrow-\infty} \Delta_{\mu_{3}}(k ; z)=1$ существует $z_{1}<-m$ такое, что $\Delta_{\mu_{3}}\left(k ; z_{1}\right)>0$. Поскольку $\Delta_{\mu_{3}}(k ; z)$ является непрерывной функцией (по $z$ ) и на концах сегмента $\left[z_{1},-m\right]$ принимает значения разных знаков, она обращается в нуль в некоторой точке $z_{3}(k) \in\left(z_{1},-m\right)$, которая в силу монотонности единственна. Следовательно, оператор $h_{3}(k)$ имеет единственное собственное значение, меньшее $-m$.

2. Отметим, что

$$
\max _{k} \int_{\mathbb{T}^{3}} \frac{d q}{\varepsilon_{\alpha}(k ; q)-z}=\int_{\mathbb{T}^{3}} \frac{d q}{\varepsilon_{\alpha}(\mathbf{0} ; q)-z}<\int_{\mathbb{T}^{3}} \frac{d q}{\varepsilon_{\alpha}(\mathbf{0} ; q)}=\frac{1}{\mu_{\alpha}^{0}}, \quad z<0 .
$$

Поэтому для всех $k \in \mathbb{T}^{3}$ и $z<0$ имеем $\Delta_{\mu_{\alpha}}(k ; z) \geqslant \Delta_{\mu_{\alpha}}(0 ; z)>1-\mu_{\alpha} / \mu_{\alpha}^{0} \geqslant 0$ при $\mu_{\alpha} \leqslant \mu_{\alpha}^{0}, \alpha=1,2$. Отсюда следует утверждение 2 .

ДОКАЗАТЕЛЬСТВО УТВЕРЖДЕНИЯ 1 теОРЕМЫ 1 . Пусть $\mu_{\alpha} \leqslant \mu_{\alpha}^{0}, \alpha=1,2$. Тогда согласно утверждению 2 леммы 7 из равенства $(2.3)$ имеем $\sigma\left(H_{\alpha}(\mathbf{0})\right)=\left[0, M_{\mathbf{0}}\right]$ при $\alpha=1,2$. Пусть $\mu_{3}>\mu_{3}^{*}$. Тогда в силу леммы 7 для каждого $k \in \mathbb{T}^{3}$ существует $z_{3}(k)<-m$ такое, что $\Delta_{\mu_{3}}\left(k ; z_{3}(k)\right)=0$. Функция $\Delta_{\mu_{3}}(\cdot ; z)$ является дифференцируемой в $\mathbb{T}^{3}$, поэтому $z_{3}(k)$ является непрерывной в $\mathbb{T}^{3}$. Отсюда имеем $\bigcup_{p \in \mathbb{T}^{3}} \sigma_{\mathrm{disc}}\left(h_{3}(p)\right)=\left[a_{3}, b_{3}\right]$, где $a_{3}=\inf _{p} z_{3}(p), b_{3}=\sup _{p} z_{3}(p)<-m$. Согласно равенству $(2.3)$ получаем $\sigma\left(H_{3}(\mathbf{0})\right)=\left[0, M_{\mathbf{0}}\right] \cup\left[a_{3}, b_{3}\right]$. В результате из леммы 1 имеем $\sigma_{\text {ess }}(H(\mathbf{0}))=\left[0, M_{\mathbf{0}}\right] \cup\left[a_{3}, b_{3}\right]$.

Ниже мы докажем утверждение 2 теоремы 1.

В работе [10] с учетом невырожденности точки минимума для функции $\varepsilon(\cdot)$ и с помощью леммы Морса после некоторых вычислений доказывается

Лемма 8. Пусть $\mu_{3}>\mu_{3}^{*}, \mu_{\alpha}=\mu_{\alpha}^{0}, \alpha=1,2$. Тогда существует число $\delta>0$ такое, что для любых $p_{\alpha} \in U_{\delta}(\mathbf{0})=\left\{p \in \mathbb{T}^{3}:|p|<\delta\right\}$ функция $\Delta_{\mu_{\alpha}}\left(p_{\alpha} ; z-\varepsilon_{\alpha}\left(p_{\alpha}\right)\right)$ представляется в виде

$$
\Delta_{\mu_{\alpha}}\left(p_{\alpha} ; z-\varepsilon_{\alpha}\left(p_{\alpha}\right)\right)=\frac{\mu_{\alpha}}{\sqrt{2} \pi} l_{\alpha}^{3 / 2} \sqrt{\frac{1}{2 n_{\alpha}} p_{\alpha}^{2}-z}+O\left(\left|p_{\alpha}\right|^{2}\right)+o(|z|), \quad|z| \rightarrow 0,
$$

где $l_{\alpha}=m_{\alpha} m_{\beta} /\left(m_{\beta}+m_{\gamma}\right), n_{\alpha}=m_{\alpha}\left(m_{\beta}+m_{\gamma}\right) /\left(m_{1}+m_{2}+m_{3}\right)$.

Лемма 9. Пусть $\mu_{3}>\mu_{3}^{*}, \mu_{\alpha}=\mu_{\alpha}^{0}, \alpha=1,2$. Тогда операторнозначные функиии $T_{\alpha \beta}(z), \alpha, \beta=1,2$, являются непрерывными в смысле операторной топологии в $\left(b_{3}, 0\right) u \lim _{z \rightarrow-0} T_{\alpha \beta}(z)=T_{\alpha \beta}(0), \alpha, \beta=1,2$. 
ДокАЗАТЕЛьСтво. Поскольку $\mu_{3}>\mu_{3}^{*}$, согласно лемме $7 \sigma\left(H_{3}(\mathbf{0})\right)=\left[a_{3}, b_{3}\right] \cup$ $\left[0, M_{\mathbf{0}}\right], b_{3}<0$. Поэтому для каждого $p \in \mathbb{T}^{3}$ существует $z_{0} \in\left[a_{3}, b_{3}\right]$ такое, что $\Delta_{\mu_{3}}\left(\mathbf{0}-p ; z_{0}-\left(1 / m_{3}\right) \varepsilon(p)\right)=0$. Из монотонности функции $\Delta_{\mu_{3}}\left(\mathbf{0}-p ; z-\left(1 / m_{3}\right) \varepsilon(p)\right)$ по $z \in(-\infty, 0)$ вытекает, что существует $\delta>0$ такое, что для всех $p \in \mathbb{T}^{3}$ имеет место неравенство $\Delta_{\mu_{3}}\left(\mathbf{0}-p ; 0-\left(1 / m_{3}\right) \varepsilon(p)\right) \neq 0$, т.е. оператор $W_{3}(\mathbf{0})$ является ограниченным.

Поэтому для доказательства леммы достаточно показать квадратичную интегрируемость ядра интегрального оператора $W_{\alpha}^{1 / 2}(z) K_{\alpha 3}(z)$ для всех $z \in\left[b_{3} / 2,0\right]$ и $\alpha=1,2$. Функция $\mathcal{E}_{\mathbf{0}}(\cdot, \cdot)$ достигает своего минимума только в точке $(\mathbf{0}, \mathbf{0}) \in\left(\mathbb{T}^{3}\right)^{2}$, и эта точка является невырожденной, поэтому для всех $p, p^{\prime} \in\left(\mathbb{T}^{3}\right)^{2}, z \in\left[b_{3} / 2,0\right]$ имеет место неравенство

$$
\left|K_{\alpha 3}\left(z ; p, p^{\prime}\right)\right| \leqslant \frac{C}{p^{2}+p^{2}}, \quad \alpha=1,2 .
$$

Следовательно, согласно лемме 8 для всех $p, p^{\prime} \in\left(\mathbb{T}^{3}\right)^{2}$ и $z \in\left[b_{3} / 2,0\right]$ получаем

$$
\left|\frac{1}{\Delta_{\mu_{\alpha}}\left(p ; 0-\left(1 / m_{3}\right) \varepsilon(p)\right)} K_{\alpha 3}\left(z ; p, p^{\prime}\right)\right| \leqslant \frac{C^{\prime}}{\sqrt{|p|}} \frac{1}{p^{2}+p^{\prime 2}}, \quad \alpha=1,2 .
$$

Отсюда получаем квадратичную интегрируемость ядра интегрального оператора $W_{\alpha}^{1 / 2}(z) K_{\alpha 3}(z)$ для всех $z \in\left[b_{3} / 2,0\right]$ и $\alpha=1,2$.

Из леммы 9 вытекает

Лемма 10. Пусть $\mu_{3}>\mu_{3}^{*}, \mu_{\alpha}=\mu_{\alpha}^{0}, \alpha=1,2$. Для каждого $z<0$ оператор $\mathbf{T}(z)-\mathbf{T}_{0}(z)$ является компактным и оператор-функиия $\mathbf{T}(z)-\mathbf{T}_{0}(z)$ непрерывна слева в точке $z=0$, где

$$
\mathbf{T}_{0}(z)=\left(\begin{array}{cc}
0 & W_{1}^{1 / 2}(z) K_{12}(z) W_{2}^{1 / 2}(z) \\
W_{2}^{1 / 2}(z) K_{21}(z) W_{1}^{1 / 2}(z) & 0
\end{array}\right) .
$$

Отметим, что $W_{1}^{1 / 2}(z) K_{12}(z) W_{2}^{1 / 2}(z)=\left[W_{2}^{1 / 2}(z) K_{21}(z) W_{1}^{1 / 2}(z)\right]^{*}$ и ядро интегрального оператора $W_{1}^{1 / 2}(z) K_{12}(z) W_{2}^{1 / 2}(z)$ имеет вид

$$
T_{12}(z ; p, q)=\frac{\sqrt{\mu_{1} \mu_{2}}(2 \pi)^{-3}\left(\mathcal{E}_{\mathbf{0}}(p, q)-z\right)^{-1}}{\sqrt{\Delta_{\mu_{1}}\left(p ; z-\varepsilon_{1}(p)\right)} \sqrt{\Delta_{\mu_{2}}\left(q ; z-\varepsilon_{2}(q)\right)}} .
$$

Пусть $\Omega_{\rho}(z)=\left\{p \in \mathbb{T}^{3}:|z|^{1 / 2} \rho \leqslant|p| \leqslant \rho\right\}, \chi_{\rho}(p)$ - характеристическая функция шара $\Omega_{\rho}(z) \subset \mathbb{T}^{3}, \mathbf{T}_{\rho}(z)$ - оператор, действующий в пространстве $L_{2}^{(2)}\left(\mathbb{T}^{3}\right)$ с элементами $t_{11}(z)=t_{22}(z)=0, t_{12}(z)=t_{21}^{*}(z)$, где $t_{12}(z)$ - интегральный оператор, порожденный ядром

$$
t_{12}(z ; p, s)=\sqrt[4]{\frac{n_{1} n_{2}}{l_{1}^{3} l_{2}^{3}}} \frac{1}{2 \pi^{2}} \frac{\chi_{\rho}(p) \chi_{\rho}(s)}{|p|^{1 / 2}\left(p^{2} / l_{2}+2 p s / m_{3}+s^{2} / l_{1}\right)|s|^{1 / 2}} .
$$

Лемма 11. Для каждого $z \in\left(b_{3}, 0\right)$ оператор $\mathbf{T}_{0}(z)-\mathbf{T}_{\rho}(z)$ является компактным и оператор-функция $\mathbf{T}_{0}(z)-\mathbf{T}_{\rho}(z)$ непрерьвна слева в точке $z=0$. 
ДокАЗАтЕльство. Достаточно показать, что оператор $W_{1}^{1 / 2}(z) K_{12}(z) W_{2}^{1 / 2}(z)-$ $t_{12}(z)$ принадлежит классу Гильберта-Шмидта, т.е. при каждом $z \in\left(b_{3}, 0\right]$ сходится интеграл

$$
I(z)=\int_{\mathbb{T}^{3}} \int_{\mathbb{T}^{3}}|D(p, q ; z)|^{2} d p d q, \quad D(p, q ; z)=T_{12}(z ; p, q)-t_{12}(z ; p, q) .
$$

Представим $I(z)$ в виде

$$
I(z)=\sum_{i, j=1}^{2} \int_{A_{i}} \int_{A_{j}}|D(p, q ; z)|^{2} d p d q,
$$

где $A_{1}=\mathbb{T}^{3} \backslash \Omega_{\rho}(z), A_{2}=\Omega_{\rho}(z)$.

Докажем интегрируемость функции $|D(p, q ; z)|^{2}$ на множествах $A_{1} \times A_{1}$ и $A_{2} \times A_{2}$, содержащих особенности функции $|D(p, q ; 0)|$ более высокого порядка. Запишем функцию $D(p, q ; z)$ в виде суммы трех функций: $D(p, q ; z)=D_{1}(p, q ; z)+D_{2}(p, q ; z)+$ $D_{3}(p, q ; z)$, где

$$
\begin{aligned}
D_{1}(p, q ; z)= & \frac{1}{2 \pi^{2}}\left[\frac{\sqrt{\mu_{1}}(2 \pi)^{-1 / 2}}{\sqrt{\Delta_{\mu_{1}}\left(p ; z-\varepsilon_{1}(p)\right)}}-\sqrt[4]{\frac{n_{1}}{l_{1}^{3}}} \frac{1}{|p|}\right] \times \\
& \times \frac{1}{2\left(\mathcal{E}_{\mathbf{0}}(p, q)-z\right)} \frac{\sqrt{\mu_{2}}(2 \pi)^{-1 / 2}}{\sqrt{\Delta_{\mu_{2}}\left(q ; z-\varepsilon_{2}(q)\right)}}, \\
D_{2}(p, q ; z)= & \frac{1}{2 \pi^{2}} \sqrt[4]{\frac{n_{1}}{l_{1}^{3}}} \frac{1}{|p|^{1 / 2}}\left[\frac{1}{2\left(\mathcal{E}_{0}(p, q)-z\right)}-\frac{1}{p^{2} / l_{2}+2 p q / m_{3}+q^{2} / l_{1}}\right] \times \\
& \times \frac{\sqrt{\mu_{2}}(2 \pi)^{-1 / 2}}{\sqrt{\Delta_{\mu_{2}}\left(q ; z-\varepsilon_{2}(q)\right)}}, \\
D_{3}(p, q ; z)= & \frac{1}{2 \pi^{2}} \sqrt[4]{\frac{n_{1}}{l_{1}^{3}}} \frac{1}{|p|^{1 / 2}} \times \\
& \times \frac{1}{p^{2} / l_{2}+2 p q / m_{3}+q^{2} / l_{1}}\left[\frac{\sqrt{\mu_{2}}(2 \pi)^{-1 / 2}}{\sqrt{\Delta_{\mu_{2}}\left(q ; z-\varepsilon_{2}(q)\right)}}-\sqrt[4]{\frac{n_{2}}{l_{2}^{3}}} \frac{1}{|q|^{1 / 2}}\right] .
\end{aligned}
$$

Согласно лемме 8 из равенства

$$
\begin{aligned}
J\left(p_{\alpha} ; z\right) & =: \frac{\sqrt{\mu_{\alpha}}(2 \pi)^{-1 / 2}}{\sqrt{\Delta_{\mu_{\alpha}}\left(p_{\alpha} ; z-\varepsilon_{2}\left(p_{\alpha}\right)\right)}}-\sqrt[4]{\frac{n_{\alpha}}{l_{\alpha}^{3}}} \frac{1}{\left|p_{\alpha}\right|^{1 / 2}}= \\
& =\frac{\sqrt{\mu_{\alpha}}(2 \pi)^{-1 / 2} \sqrt[4]{l_{\alpha}^{3} / n_{\alpha}}\left|p_{\alpha}\right|^{1 / 2}-\sqrt{\Delta_{\mu_{\alpha}}\left(p_{\alpha} ; z-\varepsilon_{2}\left(p_{\alpha}\right)\right)}}{\sqrt{\Delta_{\mu_{\alpha}}\left(p_{\alpha} ; z-\varepsilon_{2}\left(p_{\alpha}\right)\right)} \sqrt[4]{l_{\alpha}^{3} / n_{\alpha}}\left|p_{\alpha}\right|^{1 / 2}}
\end{aligned}
$$

для всех $p_{\alpha} \in \Omega_{\rho}(z)$ при $z \leqslant 0$ получаем ограниченность $\left|J\left(p_{\alpha} ; z\right)\right|$ :

$$
\begin{aligned}
\left|J\left(p_{\alpha} ; z\right)\right| \leqslant & \frac{C}{\sqrt{\Delta_{\mu_{\alpha}}\left(p_{\alpha} ; z-\varepsilon_{2}\left(p_{\alpha}\right)\right)}\left|p_{\alpha}\right|^{1 / 2}} \frac{O\left(p_{\alpha}^{2}\right)-z}{\sqrt{\Delta_{\mu_{\alpha}}\left(p_{\alpha} ; z-\varepsilon_{2}\left(p_{\alpha}\right)\right)}+\left|p_{\alpha}\right|^{1 / 2}} \leqslant \\
\leqslant & \frac{O\left(p_{\alpha}^{2}\right)-z}{\left|p_{\alpha}\right|^{3 / 2}} \frac{C^{\prime}}{\sqrt{\Delta_{\mu_{\alpha}}\left(p_{\alpha} ; z-\varepsilon_{2}\left(p_{\alpha}\right)\right) /\left|p_{\alpha}\right|}} \times \\
& \times \frac{1}{\sqrt{\Delta_{\mu_{\alpha}}\left(p_{\alpha} ; z-\varepsilon_{2}\left(p_{\alpha}\right)\right) /\left|p_{\alpha}\right|}+1} \leqslant C^{\prime \prime}, \quad \alpha=1,2 .
\end{aligned}
$$


Отсюда следует сходимость интеграла

$$
\int_{A_{2}} \int_{A_{2}}\left|D_{1}(p, q ; z)\right|^{2} d p d q, \quad z \leqslant 0 .
$$

Сходимость интеграла

$$
\int_{A_{2}} \int_{A_{2}}\left|D_{2}(p, q ; z)\right|^{2} d p d q, \quad z \leqslant 0,
$$

доказывается аналогично.

Из невырожденности точки минимума функции $\mathcal{E}_{\mathbf{0}}(\cdot, \cdot)$ вытекает неравенство

$$
\left|\frac{h_{3}(p, s)}{\mathcal{E}_{0}(p, q)-z} \frac{1}{p^{2} / l_{2}+2 p q / m_{3}+q^{2} / l_{1}}\right| \leqslant C,
$$

где

$$
\begin{gathered}
h_{3}(p, s)=\mathcal{E}_{0}(p, q)-\frac{p^{2}}{l_{2}}+\frac{2 p q}{m_{3}}+\frac{q^{2}}{l_{1}}=\sum_{|l|+|n| \geqslant 4} C_{l n} p^{l} q^{n} \\
p^{s}=p_{1}^{s_{1}} p_{2}^{s_{2}} p_{3}^{s_{3}}, \quad|s|=s_{1}+s_{2}+s_{3} .
\end{gathered}
$$

Поэтому для квадратичной интегрируемости функции

$$
D_{2}(p, q ; z)=\sqrt[4]{\frac{n_{1}}{l_{1}^{3}}} \frac{1}{|p|^{1 / 2}} \frac{h_{3}(p, s)+z}{\mathcal{E}_{0}(p, q)-z} \frac{1}{p^{2} / l_{2}+2 p q / m_{3}+q^{2} / l_{1}} \frac{1}{\sqrt{\Delta_{\mu_{2}}\left(q ; z-\varepsilon_{2}(q)\right)}}
$$

на множестве $A_{2} \times A_{2}$ достаточно сходимости интеграла

$$
J(z)=\int_{A_{2}} \int_{A_{2}} \frac{1}{|p|} \frac{z^{2}}{\left[\mathcal{E}_{0}(p, q)-z\right]^{2}} \frac{d p}{\left[p^{2} / l_{2}+2 p q / m_{3}+q^{2} / l_{1}\right]^{2}} \frac{d q}{\Delta_{\mu_{2}}\left(q ; z-\varepsilon_{2}(q)\right)} .
$$

Используя лемму 8 для всех $p, q \in \mathbb{T}^{3}$, получаем оценку

$$
\begin{aligned}
& \frac{1}{\left[\mathcal{E}_{0}(p, q)-z\right]^{2}} \frac{1}{\left[p^{2} / l_{2}+2 p q / m_{3}+q^{2} / l_{1}\right]^{2}} \frac{1}{\Delta_{\mu_{2}}\left(q ; z-\varepsilon_{2}(q)\right)} \leqslant \\
& \leqslant \frac{1}{\left[p^{2}+q^{2}-z\right]^{2}} \frac{C}{\left[p^{2}+q^{2}\right]^{2}} \frac{1}{|q|} .
\end{aligned}
$$

Переходя к сферическим координатам, получаем

$$
\begin{aligned}
J(z) & \leqslant \int_{A_{2}} \int_{A_{2}} \frac{z^{2}}{|p|} \frac{d p}{\left[p^{2}+q^{2}-z\right]^{2}} \frac{d q}{\left[p^{2}+q^{2}\right]^{2}} \frac{1}{|q|} \leqslant \\
& \leqslant \int_{\rho|z| / 2}^{\rho} \int_{\rho|z| / 2}^{\rho} \frac{C^{\prime} z^{2} d r}{\left[r^{2}+\rho^{2}-z\right]^{2}} \frac{d \rho}{r^{2}+\rho^{2}}<C^{\prime \prime} \int_{\rho|z| / 2}^{\sqrt{2} \rho} \frac{z d t}{\left(t^{2}-z\right) t}<\infty .
\end{aligned}
$$

Точно так же

$$
\begin{aligned}
\int_{A_{1}} \int_{A_{1}}|D(p, q ; z)|^{2} d p d q & =\int_{A_{1}} \int_{A_{1}}\left|T_{12}(z ; p, q)\right|^{2} d p d q= \\
& =\sum_{i, j=1}^{2} \int_{B_{i}} \int_{B_{j}}\left|T_{12}(z ; p, q)\right|^{2} d p d q
\end{aligned}
$$


где $B_{1}=\mathbb{T}^{3} \backslash U_{\rho}(\mathbf{0}), B_{2}=U_{\rho|z| / 2}(\mathbf{0}), U_{r}(\mathbf{0})=\{p:|p|<r\}$. Элементарное рассуждение показывает, что

$$
\begin{aligned}
\int_{B_{2}} \int_{B_{2}}\left|T_{12}(z ; p, q)\right|^{2} d p d q & \leqslant C \int_{B_{2}} \int_{B_{2}} \frac{1}{|p|} \frac{d p}{\left[p^{2}+q^{2}-z\right]^{2}} \frac{d q}{|q|} d p d q \leqslant \\
& \leqslant C^{\prime} \int_{0}^{\rho|z| / 2} \int_{0}^{\rho|z| / 2} \frac{r \rho d r d \rho}{\left[r^{2}+\rho^{2}-z\right]^{2}}<\infty
\end{aligned}
$$

Сходимость интегралов

$$
\begin{gathered}
\int_{B_{1}} \int_{B_{1}}\left|T_{12}(z ; p, q)\right|^{2} d p d q, \quad \int_{B_{1}} \int_{B_{2}}\left|T_{12}(z ; p, q)\right|^{2} d p d q \\
\int_{B_{2}} \int_{B_{1}}\left|T_{12}(z ; p, q)\right|^{2} d p d q \\
\int_{A_{1}} \int_{A_{2}}|D(p, q ; z)|^{2} d p d q, \quad \int_{A_{2}} \int_{A_{1}}|D(p, q ; z)|^{2} d p d q
\end{gathered}
$$

доказывается аналогично.

Пространство всех векторов $(\varphi, \psi) \in L_{2}^{(2)}\left(\mathbb{T}^{3}\right)$, носители компонент которых лежат в $\Omega_{\rho}(z)$, является инвариантным подпространством оператора $\mathbf{T}_{\rho}(z)$. Пусть $\widehat{\mathbf{T}}_{\rho}(z)-$ сужение оператора $\mathbf{T}_{\rho}(z)$ на это подпространство.

Введем унитарный оператор

$$
\begin{gathered}
U: L_{2}\left(\Omega_{\rho}(z)\right) \rightarrow L_{2}\left((0, R), L_{2}\left(\mathbb{S}^{2}\right)\right), \\
(U f)(x, \xi)=\left(|z|^{1 / 2} \rho\right)^{3 / 2} e^{3 x / 2} f\left(|z|^{1 / 2} \rho e^{x} \xi\right), \quad x \in(0, R), \quad \xi \in \mathbb{S}^{2},
\end{gathered}
$$

где $R=-(\ln |z|) / 2, \mathbb{S}^{2}$ - единичная сфера, при этом

$$
U^{-1}: L_{2}\left((0, R), L_{2}\left(\mathbb{S}^{2}\right)\right) \rightarrow L_{2}\left(\Omega_{\rho}(z)\right), \quad\left(U^{-1} f\right)(p)=|p|^{-3 / 2} f\left(\ln \frac{|p|}{|z|^{1 / 2} \rho}, \frac{p}{|p|}\right) .
$$

Обозначим через $\mathbf{S}_{R}$ матричнозначный оператор, действующий в пространстве $L_{2}^{(2)}\left((0, R), L_{2}\left(\mathbb{S}^{2}\right)\right)$, с элементами $S_{11}(z)=S_{22}(z)=0, S_{12}(z)=S_{21}^{*}(z)$, где $S_{12}(z)-$ интегральный оператор, определенный в пространстве $L_{2}\left((0, R), L_{2}\left(\mathbb{S}^{2}\right)\right)$ как

$$
\begin{aligned}
\left(S_{12} f\right)(x ; \xi) & =\int_{0}^{R} \int_{\mathbb{S}^{2}} S_{12}(x-y ;(\xi, \eta)) f(y, \eta) d y d \eta \\
S_{12}(x ; t) & =\sqrt[4]{\frac{n_{1} n_{2}}{l_{1}^{3} l_{2}^{3}}} \frac{1}{2 \pi^{2}} \frac{1}{2 / \sqrt{l_{1} l_{2}} \operatorname{ch}\left(x+\left(\ln \left(l_{2} / l_{1}\right)\right) / 2\right)+2 t / m_{3}}
\end{aligned}
$$

$(\cdot, \cdot)$ - скалярное произведение в $\mathbb{S}^{2}$.

Оператор $\widehat{\mathbf{T}}_{\rho}(z)$ унитарно эквивалентен оператору $\mathbf{S}_{R}$. Эквивалентность осуществляется с помощью унитарного оператора $\mathbf{U}=\left(\begin{array}{cc}U & 0 \\ 0 & U\end{array}\right)$, т.е. $\mathbf{S}_{R}=\mathbf{U} \widehat{\mathbf{T}}_{\rho}(z) \mathbf{U}^{-\mathbf{1}}$.

Обозначим через $L^{(l)}\left(\mathbb{S}^{2}\right) \subset L_{2}\left(\mathbb{S}^{2}\right), l=0,1,2, \ldots$, подпространство функций с угловым моментом $l, \operatorname{dim} L^{(l)}\left(\mathbb{S}^{2}\right)=2 l+1$ (см. [12]),

$$
L_{2}\left(\mathbb{S}^{2}\right)=\sum_{l} \oplus L^{(l)}\left(\mathbb{S}^{2}\right)
$$


Пусть $\widehat{P}_{l}: L_{2}\left(\mathbb{S}^{2}\right) \rightarrow L^{(l)}\left(\mathbb{S}^{2}\right)$ - ортогональный проектор на $L^{(l)}\left(\mathbb{S}^{2}\right)$. Ядро оператора $\widehat{P}_{l}$ выражается через многочлен Лежандра $P_{l}$ :

$$
\widehat{P}_{l}((\xi, \eta))=\frac{2 l+1}{4 \pi} P_{l}((\xi, \eta)) .
$$

Ядро оператора $\mathbf{S}_{R}$ на сфере зависит только от скалярного произведения $(\xi, \eta)$, поэтому подпространство $\mathbb{C}^{2} \otimes L^{(l)}\left(\mathbb{S}^{2}\right)$ является инвариантным относительно оператора $\mathbf{S}_{R}$ и

$$
\mathbf{S}_{R}=\sum_{l} \oplus\left(\mathbf{S}_{R}^{(l)} \otimes \widehat{\mathbf{P}}_{l}\right), \quad \widehat{\mathbf{P}}_{l}=\left(\begin{array}{cc}
\widehat{P}_{l} & 0 \\
0 & \widehat{P}_{l}
\end{array}\right)
$$

где $\mathbf{S}_{R}^{(l)}$ - матричнозначный оператор, действующий в $L_{2}^{(2)}(0, R)$, с элементами $S_{11}^{(l)}=$ $S_{22}^{(l)}=0, S_{12}^{(l)}=\left[S_{21}^{(l)}\right]^{*}, S_{12}^{(l)}$ - интегральный оператор в $L_{2}(0, R)$, порожденный ядром

$$
S_{12}^{(l)}\left(x-x^{\prime}\right)=2 \pi \int_{-1}^{1} P_{l}(t) S_{12}\left(x-x^{\prime} ; t\right) d t
$$

при этом

$$
\sigma\left(\mathbf{S}_{R}\right)=\bigcup_{l} \sigma\left(\mathbf{S}_{R}^{(l)} \otimes \widehat{\mathbf{P}}_{l}\right)=\bigcup_{l} \sigma\left(\mathbf{S}_{R}^{(l)}\right)
$$

Пусть $\mathbf{L}(z)$ - оператор, действующий в $L_{2}^{(2)}(0, R)$, заданный матрицей с элементами $L_{11}(z)=L_{22}(z)=0, L_{12}(z)=\left[L_{21}(z)\right]^{*}$, где $L_{12}(z)$ - интегральный оператор в $L_{2}(0, R)$, порожденный ядром

$$
L_{12}^{(l)}\left(x-x^{\prime}\right)=\chi_{(0, R]}\left(x-x^{\prime}\right) S_{12}^{(0)}\left(x-x^{\prime}\right) .
$$

ЛЕмма 12. Для каждого $z<0$ оператор $\mathbf{S}_{R}^{(0)}-\mathbf{L}(z)$ является компактным и $\left\|\mathbf{S}_{R}^{(0)}-\mathbf{L}(z)\right\| \rightarrow 0$ nри $z \rightarrow-0$.

ДокАЗАТЕЛЬСтво. Пользуясь неравенством Шварца и определением характеристической функции, имеем

$$
\begin{aligned}
& \left\|\left[\mathbf{S}_{R}^{(0)}(z)-\mathbf{L}(z)\right] f\right\|^{2}= \\
& \quad=\int_{0}^{R}\left|\sqrt[4]{\frac{n_{1} n_{2}}{l_{1}^{3} l_{2}^{3}}} \frac{1}{\pi} \int_{0}^{R} \int_{-1}^{1} \frac{d t[1-\chi(x-s)] f(s) d s}{\left(2 / \sqrt{l_{1} l_{2}}\right) \operatorname{ch}\left(x-s+\left(\ln \left(l_{2} / l_{1}\right)\right) / 2\right)+2 t / m_{3}}\right|^{2} d x \leqslant \\
& \quad \leqslant \frac{C R^{2}\|f\|^{2}}{\left[\operatorname{ch}\left(R+\left(\ln \left(l_{2} / l_{1}\right)\right) / 2\right)\right]^{2}} .
\end{aligned}
$$

Следовательно,

$$
\left\|\mathbf{S}_{R}^{(0)}(z)-\mathbf{L}(z)\right\| \rightarrow 0, \quad z \rightarrow 0 .
$$

Введем унитарный оператор

$$
U_{1}^{-1}: L_{2}(-\pi, \pi) \rightarrow L_{2}(0, R), \quad\left(U_{1}^{-1} f\right)(x)=\sqrt{\frac{2 \pi}{R}} f\left(\frac{2 \pi}{R} x-\pi\right) .
$$


Очевидно, что

$$
U_{1}: L_{2}(0, R) \rightarrow L_{2}(-\pi, \pi), \quad\left(U_{1} g\right)(x)=\sqrt{\frac{R}{2 \pi}} g\left(\frac{R}{2 \pi}(x+\pi)\right) .
$$

Пусть $\mathcal{L}(z)$ - матричный оператор в $L_{2}^{(2)}(-\pi, \pi]$ с элементами $\mathcal{L}_{11}(z)=\mathcal{L}_{22}(z)=0$, $\mathcal{L}_{12}(z)=\left[\mathcal{L}_{21}(z)\right]^{*}$, где $\mathcal{L}_{12}(z)-$ интегральный оператор в $L_{2}(-\pi, \pi]$, порожденный ядром

$$
\mathcal{L}_{12}\left(x-x^{\prime} ; t\right)=\sqrt[4]{\frac{n_{1} n_{2}}{l_{1}^{3} l_{2}^{3}}} \frac{R}{2 \pi} \frac{1}{\pi} \int_{-1}^{1} \frac{d t}{\left(2 / \sqrt{l_{1} l_{2}}\right) \operatorname{ch}\left(R\left(x-x^{\prime}\right) /(2 \pi)+\left(\ln \left(l_{2} / l_{1}\right)\right) / 2\right)+2 t / m_{3}} .
$$

Оператор $\mathcal{L}(z)$ унитарно эквивалентен оператору $\mathbf{L}(z)$, эквивалентность определяется унитарным оператором $\mathbf{U}_{1}=\left(\begin{array}{cc}U_{1} & 0 \\ 0 & U_{1}\end{array}\right)$, т.е. $\mathcal{L}(z)=\mathbf{U}_{1} \mathbf{L}(z) \mathbf{U}_{1}^{-1}$.

Если $\lambda \neq 0$ - собственное число оператора $\mathcal{L}(z)$, то оно имеет вид $\lambda= \pm\left|u_{n}(z)\right|$ и $\Phi^{ \pm}= \pm\left(f_{n}, \frac{\overline{u_{n}(z)}}{\left|u_{n}(z)\right|} f_{n}\right)$ является соответствующим собственным вектором, где $f_{n}(x)=$ $e^{i n x} / \sqrt{2 \pi}, n=0, \pm 1, \pm 2, \ldots$,

$$
u_{n}(z)=\sqrt[4]{\frac{n_{1} n_{2}}{l_{1}^{3} l_{2}^{3}}} \frac{R}{2 \pi} \frac{1}{\pi} \int_{-\pi}^{\pi} \int_{-1}^{1} \frac{e^{i n s} d t d s}{\left(2 / \sqrt{l_{1} l_{2}}\right) \operatorname{ch}\left(R s /(2 \pi)+\left(\ln \left(l_{2} / l_{1}\right)\right) / 2\right)+2 t / m_{3}} .
$$

Поэтому получаем

$$
\sigma(\mathbf{L}(z))=\sigma(\mathcal{L}(z))=\bigcup_{n \in \mathbb{Z}}\left\{\left|u_{n}(z)\right|\right\} \cup\left\{-\left|u_{n}(z)\right|\right\} \cup\{0\}, \quad z<0 .
$$

Лемма 13. Имеет место равенство

$$
\left|u_{n}(z)\right|=F\left(\frac{4 \pi n}{|\ln | z||}\right)+O\left(|z|^{1 / 4}|\ln (-z)|\right), \quad z \rightarrow-0
$$

¿əe

$$
F(x)=\sqrt[4]{\frac{n_{1} n_{2}}{l_{1}^{3} l_{2}^{3}}} \frac{m_{3}}{x} \frac{\operatorname{sh}\left(x \arcsin \left(\sqrt{l_{1} l_{2}} / m_{3}\right)\right)}{\operatorname{ch}(\pi x / 2)} .
$$

ДоказАтЕльство. В силу малости $|z|$ справедливо $R / 2+\left(\ln \left(l_{2} / l_{1}\right)\right) / 2>0$. Интеграл (5.2) после замены переменной $y=R s /(2 \pi)+\left(\ln \left(l_{2} / l_{1}\right)\right) / 2$, принимает вид

$$
u_{n}(z)=e^{-(i n / 2) \ln \left(l_{2} / l_{1}\right)} \sqrt[4]{\frac{n_{1} n_{2}}{l_{1}^{3} l_{2}^{3}}}\left[I_{0}(n, z)-I_{1}(n, z)\right],
$$

где

$$
\begin{aligned}
& I_{0}(n, z)=\int_{-1}^{1} \frac{1}{\pi} \int_{-\infty}^{\infty} \frac{d t e^{i n 2 \pi y / R} d y}{2 \operatorname{ch} y / \sqrt{l_{1} l_{2}}+2 t / m_{3}} \\
& I_{1}(n, z)=\int_{-1}^{1} \frac{1}{\pi} \int_{|y|>R / 2+\left(\ln \left(l_{2} / l_{1}\right)\right) / 2} \frac{d t e^{i n 2 \pi y / R} d y}{2 \operatorname{ch} y / \sqrt{l_{1} l_{2}}+2 t / m_{3}} .
\end{aligned}
$$


Простое вычисление показывает, что для некоторого $C>0$ и для всех натуральных $n$ и $z<0$ выполняется неравенство

$$
\left|I_{1}(n, z)\right| \leqslant \frac{C R}{\operatorname{ch}(R / 2)}, \quad \frac{R}{\operatorname{ch}(R / 2)}=\frac{|z|^{1 / 4}|\ln (-z)|}{|z|^{1 / 2}+1} .
$$

Используя известное равенство (см., например, [16])

$$
\frac{1}{2 \pi} \int_{-\infty}^{\infty} \frac{\sin a e^{i w r}}{\operatorname{ch} w+\cos a} d w=\frac{\operatorname{sh} a r}{\operatorname{sh} \pi r}, \quad|a|<\pi
$$

вычисляем интеграл

$$
I_{0}(n, z)=\frac{m_{3}}{\operatorname{sh} \pi r} \int_{\arccos \left(\sqrt{l_{1} l_{2}} / m_{3}\right)}^{\pi-\arccos \left(\sqrt{l_{1} l_{2}} / m_{3}\right)} \operatorname{sh} r x d x, \quad r=\frac{2 \pi n}{R} .
$$

Учитывая элементарные равенства $\operatorname{ch} a-\operatorname{ch} b=2 \operatorname{sh}((a+b) / 2) \operatorname{sh}((a-b) / 2), \operatorname{sh} a=$ $2 \operatorname{sh}(a / 2) \operatorname{ch}(a / 2)$ и $\pi-\arccos x=\arcsin x$ при $x \in[0,1]$, получаем

$$
I_{0}(n, z)=\frac{m_{3}}{r} \frac{\operatorname{sh}\left[r \arccos \left(\sqrt{l_{1} l_{2}} / m_{3}\right)\right]}{\operatorname{ch}(\pi r / 2)} .
$$

Следовательно, $\left|I_{0}(n, z)\right|=F(4 \pi n /|\ln | z||)$.

Используя неравенство $\arcsin |x|>|x|$ при $x \in(-1,1)$, получаем

$$
F(0)=\lim _{x \rightarrow 0} F(x)=\sqrt[4]{\frac{n_{1} n_{2}}{l_{1}^{3} l_{2}^{3}}} m_{3} \arcsin \frac{\sqrt{l_{1} l_{2}}}{m_{3}}>\sqrt[4]{\frac{n_{1} n_{2}}{l_{1} l_{2}}}=\sqrt{\frac{\left(m_{1}+m_{3}\right)\left(m_{2}+m_{3}\right)}{m_{3}\left(m_{1}+m_{2}+m_{3}\right)}}>1 .
$$

Поэтому в силу монотонности функции $F$ в интервале $(0, \infty)$ существует положительное число $\delta$ такое, что уравнение $1+\delta=F(x)$ имеет единственное положительное решение $x=\lambda_{0}$. Отсюда

$$
\left|I_{0}\left(n, z_{n}\right)\right|=F\left(\frac{4 \pi n}{|\ln | z_{n}||}\right)=1+\delta, \quad z_{n}=-e^{-4 \pi n / \lambda_{0}} .
$$

Согласно лемме 13

$$
\left|u_{n}\left(z_{n}\right)\right| \geqslant 1+\delta-O\left(\left|z_{n}\right|^{1 / 4}\left|\ln \left(-z_{n}\right)\right|\right), \quad z \rightarrow-0 .
$$

Отсюда имеем

$$
\lim _{z \rightarrow-0} n(1+\delta, \mathbf{L}(z))=\lim _{z \rightarrow-0} n(1+\delta, \mathcal{L}(z))=\infty .
$$

ДОКАЗАТЕЛЬСТвО УТВЕРЖДЕНИЯ 2 тЕОРЕМЫ 1. Используя неравенство ВейЛя, получаем

$$
\begin{gathered}
n(1+\delta, \mathbf{L}(z)) \leqslant n\left(1+\frac{2 \delta}{3}, \mathbf{S}_{R}^{(0)}\right)+n\left(1+\frac{\delta}{3}, \mathbf{L}(z)-\mathbf{S}_{R}^{(0)}\right) \\
n\left(1+\frac{2 \delta}{3}, \mathbf{S}_{R}\right)=n\left(1+\frac{2 \delta}{3}, \mathbf{T}_{\rho}(z)\right) \leqslant n\left(1+\frac{\delta}{3}, \mathbf{T}_{0}(z)\right)+n\left(\frac{\delta}{3}, \mathbf{T}_{\rho}(z)-\mathbf{T}_{0}(z)\right) \\
n\left(1+\frac{\delta}{3}, \mathbf{T}_{0}(z)\right) \leqslant n(1, \mathbf{T}(z))+n\left(\frac{\delta}{3}, \mathbf{T}_{0}(z)-\mathbf{T}(z)\right)
\end{gathered}
$$


Согласно леммам 10-12 операторы $\mathbf{L}(z)-\mathbf{S}_{R}, \mathbf{T}_{\rho}(z)-\mathbf{T}_{0}(z)$ и $\mathbf{T}_{0}(z)-\mathbf{T}(z)$ являются компактными при каждом $z \in\left(b_{3}, 0\right]$. Поэтому для всех $z \in\left[b_{3} / 2,0\right]$ выполняются неравенства

$n\left(1+\frac{\delta}{3}, \mathbf{L}(z)-\mathbf{S}_{R}\right)<\infty, \quad n\left(\frac{\delta}{3}, \mathbf{T}_{\rho}(z)-\mathbf{T}_{0}(z)\right)<\infty, \quad n\left(\frac{\delta}{3}, \mathbf{T}_{0}(z)-\mathbf{T}(z)\right)<\infty$.

Отсюда, учитывая, что $n\left(1+2 \delta / 3, \mathbf{S}_{R}^{(0)}\right) \leqslant n\left(1+2 \delta / 3, \mathbf{S}_{R}\right)$, из неравенств $(5.3),(5.4)$ имеем $\lim _{z \rightarrow-0} n(1, \mathbf{T}(z))=\infty$. Согласно лемме 2 оператор $H(\mathbf{0})$ имеет бесконечное число собственных значений в интервале $\left(b_{3}, 0\right)$.

\section{Список литературы}

[1] В. Н. Ефимов, ЯФ, 12:5 (1970), 1080-1091.

[2] R. D. Amado, J. V. Noble, Phys. Rev. D, 5:8 (1971), 1992-2002.

[3] С. П. Меркурьев, Л. Д. Фаддеев, Квантовая теория рассеяния для систем нескольких частии, Наука, М., 1985.

[4] Д. Р. Яфаев, Матем. сб., 94(136):4(8) (1974), 567-593.

[5] Yu. N. Ovchinnikov, I. M. Sigal, Ann. Phys., 123:2 (1979), 274-295.

[6] H. Tamura, J. Funct. Anal., 95:2 (1991), 433-459.

[7] С. Н. Лакаев, ТМФ, 89:1 (1991), 94-104.

[8] С. Н. Лакаев, Ж. И. Абдуллаев, Матем. заметки, 71:5 (2002), 686-696.

[9] С. Н. Лакаев, М.Э. Муминов, ТМФ, 135:3 (2003), 478-503.

[10] S. Albeverio, S. N. Lakaev, Z. I. Muminov, Ann. Henri Poincaré, 5:4 (2004), 743-772.

[11] Г. М. Жислин, Труды ММО, 9, 1960, 81-120.

[12] A. V. Sobolev, Comm. Math. Phys., 156:1 (1993), 101-126.

[13] М. Рид, Б. Саймон, Методы современной математической физики. Т. 4. Анализ операторов, Мир, М., 1982.

[14] П. Халмош, Гильбертово пространство в задачах, Мир, М., 1970.

[15] М.Ш. Бирман, М.З. Соломяк, Спектральная теория самосопряженных операторов в гилъбертовом пространстве, Учеб. пособие, Изд-во ЛГУ, Л., 1980.

[16] Г. Корн, Т. Корн, Справочник по математике для научных и инженерных работников, Наука, М., 1978.

Поступила в редакцию 14.02.2008, после доработки 22.08.2008 